\title{
Awareness of mouth cancer
}

\author{
Awareness of mouth cancer in Great Britain
}

\section{R. West, M. N. Alkhatib, A. McNeill and R. Bedi Br Dent J 2006; 200: 167-169}

\section{Objective}

There are more than 2,000 new cases of mouth cancer each year in Britain. Early detection is important yet little is known about population awareness of this disease and ability to recognise early signs, particularly among high risk groups. This study aimed to address this issue.

\section{Methods}

Data were collected by means of household survey. A total of 3,384 adults were questioned using a national probability sample.

Respondents provided information on demographic characteristics, smoking status, and frequency of alcohol use. They were asked whether they had heard of mouth cancer. Their knowledge of early signs and risk factors was assessed.

\section{Results}

Whereas 95.6\% of respondents said they had heard of mouth cancer, their awareness of early signs was low; for example, only $33.8 \%$ recognised that white patches in the mouth were a sign. The large majority understood that smoking and chewing tobacco were risk factors (84.7\% and $80.1 \%$ respectively) but only $19.4 \%$ recognised alcohol use as a risk factor. In multiple logistic regression analyses controlling for relevant demographic factors, smokers and those with more frequent alcohol consumption were less likely to recognise early signs.

\section{Conclusions}

Awareness of early signs of mouth cancer is low and lower in people who as a result of their behaviour are at higher risk. There is a need to raise awareness in those at most risk.

\section{IN BRIEF}

- Mouth cancer is one of the most distressing forms of cancer because of its disfiguring effects and high mortality rate.

- The large majority of people are, in some sense, aware that they can get cancer of the mouth and that smoking is a risk factor.

- Far fewer people are aware of the role of alcohol in cancer of the mouth or what to look out for in terms of early signs.

- Those at greatest risk are generally the least likely to be aware of risk factors and early signs.

- Mass media campaigns and healthcare practitioners have an important role to play in educating those at high risk about how to reduce their risk and what to look out for in terms of early signs.

\section{COMMENT}

There is a vast literature on the subject of mouth cancer. ${ }^{1}$ It is a growing problem in the UK, with high relative mortality and increasing incidence. UK mortality in 2003 from cases of mouth cancer was higher than that for cervical cancer and similar to that for melanoma skin cancer. While members of the public can generally identify the more common symptoms such as persistent sores or ulcers and red or white patches, there is a much lower level of recognition of other symptoms such as new, persistent pains in the tongue or ear.

This paper offers the most current information on mouth cancer awareness in Great Britain's adult population and suggests that awareness of risk factors is lower in individuals at high risk because of their lifestyles. The data were collected from a large household survey of a probability sample of nearly 4,000 adults. Interviews were carried out face-to-face by trained interviewers who were instructed to make at least three attempts to interview a respondent from chosen addresses.

Four findings are especially important. While 96\% of respondents had heard of mouth cancer, awareness of early signs was low except for persistent ulcers. Female respondents were more likely to be aware of early signs. Female respondents, younger respondents, respondents of a higher occupational status and non-smoking respondents were more likely to recognise the risk factors of tobacco use and drinking alcohol. Respondents of lower occupational status were less likely to recall being counselled about mouth cancer by either their general medical or dental practitioners.

While there is an improving awareness of mouth cancer, recognition of risk factors and early signs remains similar to earlier studies. ${ }^{2}$ It is suggested that this may be due to the developing media attention given to oral cancer awareness week rather than primary health care practitioner advice. Cancer Research UK has recently launched a three year national mouth cancer awareness campaign. Targeted at older members of the population of lower occupational status who are heavy smokers and drinkers, illustrated mouth cancer referral guidelines are also being made available for medical and dental practitioners and pharmacists. A website has been set up to provide current and accessible information for both the public and health professionals. ${ }^{3}$ These separate strands of activity should be closely monitored for their impact.

Addressing the disparities in mouth cancer awareness and information giving is complex. In addition to developing public awareness there are opportunities for both undergraduate and continuing professional development. Diagnostic delays should be addressed by clear referral protocols. Clear policy initiatives will reduce the prevalence of risk factors such as smoking and excess alcohol consumption.

R. Croucher, Professor of Community Oral Health, St Bartholomew's and the Royal London School of Medicine and Dentistry

1. McLeod N M H, Saeed N R, Ali E A. Oral cancer: delays in referral and diagnosis persist. Br Dent J 2005; 198: 681-684.

2. Warnakulasuriya K A, Harris C K Scarrott D M et al. An alarming lack of public awareness towards oral cancer. Br Dent J 1999; 187: 319-322.

3. Cancer Research UK. (2005) www.openuptomouthcancer.org (accessed 30 November 2005).

doi: 10.1038/sj.bdj.4813198 